\title{
Endoscopic Stapedotomy: A New Dimension to the Conventional Microscopic Procedure
}

\author{
Bhaskar Ghosh, ${ }^{1}$ Bijan Kumar Adhikary ${ }^{1}$
}

\begin{abstract}
Introduction
Endoscopes are not only complementing microscopic ear surgery but are also establishing their independent role in recent years. Materials and Methods

This observational study was conducted from February 2018 to February 2019. Thirteen patients with clinical and audiotympanometric features suggestive of stapedial otosclerosis underwent unilateral endoscopic stapedotomy from February to July 2018. They were followed up for a minimum of 6 months, till February 2019. Zero degree naso-endoscopes were exclusively used. Details of the surgical technique, its usefulness and subjective drawbacks are described.

Results

Two patients with sequel of otitis media with effusion evident at surgery were excluded. With a female predilection, the average age of the subjects was 31 years (range: 17-56 years). Curettage of the posterosuperior wall was needed in 7 patients; significant canalplasty was performed in 2 of them where the endoscope was difficult to negotiate. The chorda tympani required mobilization in only 3 patients, including those requiring significant canalplasty. Per-operative complications included injury to the chorda, tearing of tympanomeatal flap, and instability of the long process of incus in 1 patient each. One patient was lost to follow up. Average pre-operative bone conduction was $10 \pm 5 d B$ with an air-bone gap at $35 \pm 5 d B$. At 6 months follow up, air-bone gap of $<10 \mathrm{~dB}$ was achieved in 5 patients, and that at 10-20dB in 4. One patient developed moderate sensorineural component. Otherwise, there was no significant complication at 6 months following surgery.

Conclusion

Endoscopic stapedotomy is a safe and effective approach towards stapes surgery, with better surgical field of view and minimal tissue trauma.
\end{abstract}

ABSTRACT

Keywords

Stapes Surgery; Endoscopes

$\mathrm{E}$ ndoscopic middle ear surgery is increasingly gaining ground during the last couple of decades. It not only complements microscopic ear surgery but has also attained an individual identity. In this article we share our experience in exclusive endoscopic stapes surgery and its follow up in our institute during the period of one year.

Done exclusively with a $4 \mathrm{~mm} 0^{\circ}$ nasal endoscope, a total of 13 patients were subjected to stapedotomy, who were followed up for 6-13 months. The various advantages and disadvantages of the procedure so far which we could appreciate in our initial experience are shared here.

\section{Materials and Methods}

This observational study was conducted in the department of Otorhinolaryngology and Head-Neck Surgery of a tertiary-care teaching institute of eastern India. Prior to the initiation of the study, permission from the Institutional Ethical Committee was obtained, Informed consent from the participants in the study was obtained in written form. The study period spanned for 13 months (February 2018
1 - Department of ENT, Medical College, Kolkata
Corresponding author:
Dr Bijan Kumar Adhikary
email: drbadhikary@gmail.com 


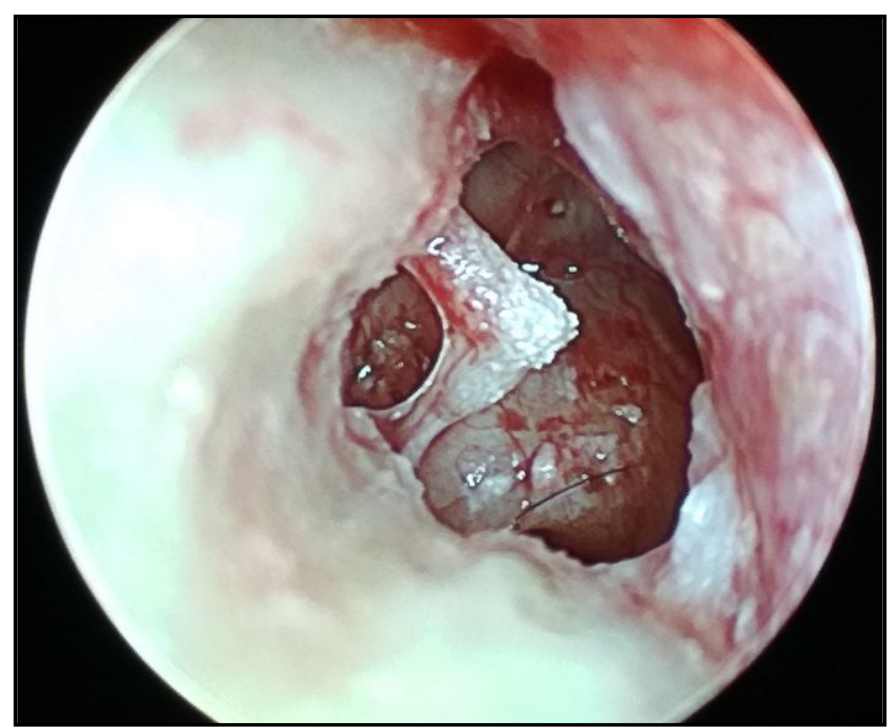

Fig. 1. Panoramic view of the middle ear with $0^{\circ}$ endoscope, following minimal curettage of the postero-superior bony overhang. Part of long process of incus, stapes suprastructure, stapedius tendon, and the footplate can be seen.

to February 2019); 13 patients were operated during the period of February 2018 to July 2018, and they were followed up for a minimum period of 6 months, that is, till February 2019, prior to writing up this report. Based on the preoperative clinical and audio-tympanometric findings, 13 patients with clinical stapedial otosclerosis were recruited for unilateral endoscopic stapedotomy; i.e., 13 ears were considered for the procedure. For the surgical procedure, $0^{\circ}$ endoscope, $18 \mathrm{~cm}$ long and $4 \mathrm{~mm}$ diameter, was exclusively used to have a panoramic view of the middle ear.

\section{Operative Technique}

On the eve of operation, we routinely performed evaluation under microscope to clean the external auditory canal (EAC) from debris, and removed hairs, where necessary, by trimming or applying hair removing gel because canal hairs are a strong deterrent for endoscopic procedures.

All the patients were operated under monitored anesthesia care. Following endomeatal incision. the tympanomeatal flap was elevated with utmost care.
Adrenaline and 4\% lignocaine soaked cottonoids were used as required to minimize bleeding. In case of a wide EAC, the chorda tympani was kept untouched, but for narrow canals with/without posterosuperior overhang, the chorda was elevated along with the flap. Once the flap was elevated, a panoramic view could be obtained with the endoscope requiring minimal or no curettage of the posterosuperior meatal wall, thereby helping to maintain the anatomic integrity. (Fig.1) After cutting the stapedial tendon and dislocating the incudostapedial joint, we cut the crura and removed the stapes suprastructure. The entire footplate could now be visualized under magnification and the stapedotomy opening could be done in a precise location. (Fig.2) We first used $0.5 \mathrm{~mm}$ perforator, and widened it with 0.6 $\mathrm{mm}$. After putting the piston in place (Fig.3), blood clot was allowed to settle around it and the tympanomeatal flap was replaced. Small tear in the flap, that occurred in one of our patients, was managed by apposing the cut margins and applying gel foam over it.

Post-operative pure tone audiometry (PTA) was advised after 6 weeks, 3 months and 6 months interval, and closure of air-bone $(\mathrm{AB})$ gaps at $500 \mathrm{~Hz}, 1000 \mathrm{~Hz}$, $2000 \mathrm{~Hz}$ and $3000 \mathrm{~Hz}$ were assessed. Patients were also evaluated for per-operative and post-operative complications during this period.

\section{Results}

Out of the 13 initial participants, 2 patients were found to have sequela of otitis media with effusion at surgery, and were excluded from the present study. The details of the study outcome are summarized in Table I. There was a female:male ratio of $8: 3$, with a definite preponderance for women in our study population. The average age of the patients was approximately 31 years (range: 17-56 years). After elevation of tympanomeatal flap, minimal curettage of posterosuperior meatal wall was required in 5 patients.

In two patients, a considerable portion was removed to allow the endoscope to be negotiated satisfactorily. In majority of the patients, 8 out of 11 , we did not have to mobilize the chorda tympani and was elevated along with the flap. However, in 3 patients, the chorda needed 


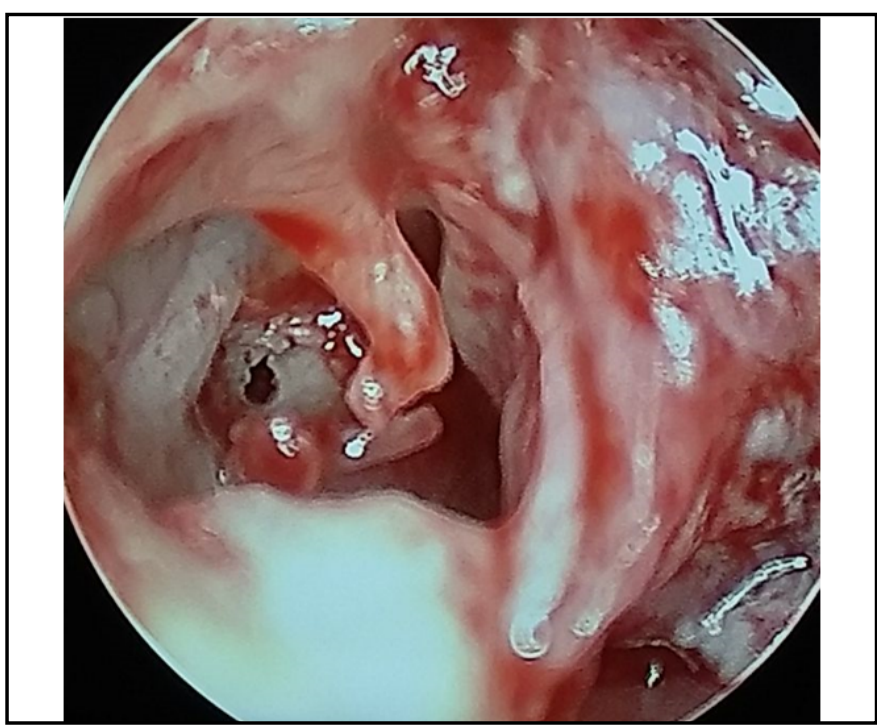

Fig. 2. The footplate can be seen clearly after removal of the stapes suprastructure. A stapedotomy has been made.

to be dissected and mobilized; in 2 of them there was significant overhanging of the posterosuperior meatal wall requiring considerable curettage.

We encountered low-lying fallopian canal in 2 ears which, unlike the endoscopic procedure, would have posed difficulty in visualization with a microscope. Peroperative complications encountered included injury to the chorda tympani (we needed to cut the nerve in 1 patient), tearing of tympanomeatal flap (1 patient), and instability/dislocation of the long process of incus (incudomalleal joint) (1 patient).

The pre-operative bone conduction in our patients was $10 \pm 5 \mathrm{~dB}$ and the $\mathrm{AB}$ gap $35 \pm 5 \mathrm{~dB}$. Post-operative average bone conduction did not change much except in 1 patient who developed mixed hearing loss within 6 weeks of surgery, with bone conduction at $35 \mathrm{~dB}$. At 6 months follow up, closure of $A B$ gap less than $10 \mathrm{~dB}$ was achieved in 5 patients and more than $10 \mathrm{~dB}$ but less than $20 \mathrm{~dB}$ in 4 . One patient was lost to follow up.

Recognized immediate post-operative complications like vertigo, nausea, vomiting and pain were minimal in our study population requiring medicines only for 1-2 days. However, the patient who developed sensorineural impairment complained of tinnitus during post-operative follow up at 6 months.

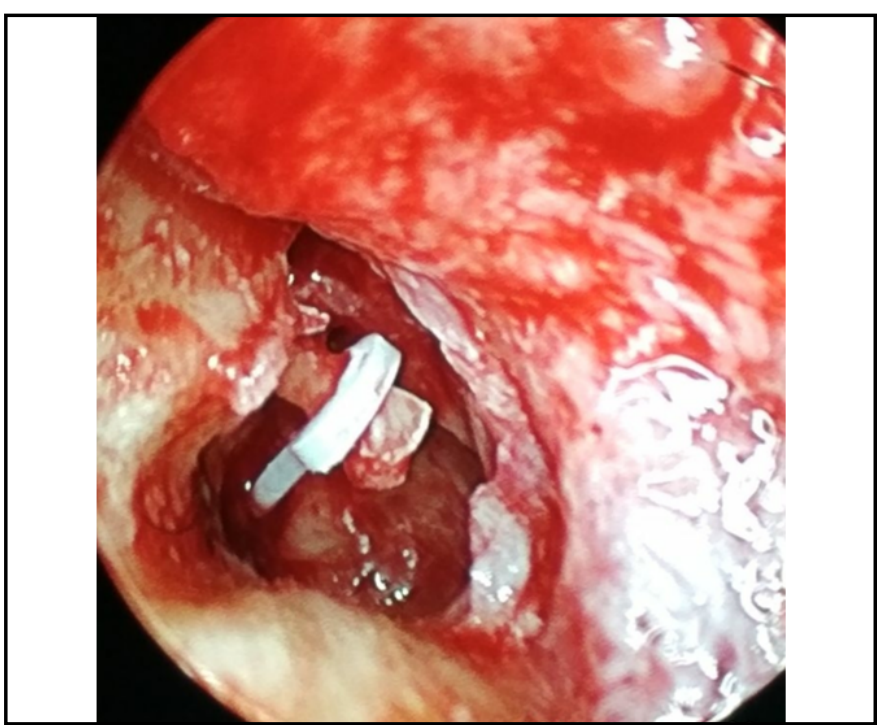

Fig. 3. Stapes piston in-situ, completing the endoscopic stapedotomy procedure.

\section{Discussion}

In the early nineties endoscopes were mostly used as diagnostic tools to complement microscopes in middle ear surgeries to see the hidden areas like facial recess, epitympanum and sinus tympani. However, since late nineties they were being increasingly used to perform middle ear surgeries, including stapes surgery. The record of the first endoscopic stapedotomy was published by Poe in 2000. ${ }^{1}$ Today, endoscopes form an integral part in most otologic and skull-base surgeries, and are often used as an exclusive visual aid for the procedure. Use of endoscopes makes the surgery minimally invasive and less traumatic ${ }^{2,3}$ as it requires only the endomeatal incision, obviating the need for the traditional endaural incision practiced widely in the eastern world owing to the relatively narrow canals compared to the Caucasians. In the truest sense, it has become a stitch-less procedure. The $4 \mathrm{~mm}, 0^{\circ}, 18 \mathrm{~cm}$ nasal endoscope gives a wide field of vision under magnification thus making the procedure much easier even in a narrow canal wall patients. ${ }^{4,5}$

Elevation of tympanomeatal flap consumes the major portion of the operative time because it is a singlehanded procedure and needs to be done with utmost care to prevent tearing. ${ }^{3}$ Once the flap is elevated, the panoramic view of the incudostapedial joint, crura of 
Table I: Details of the outcome in the endoscopic stapedotomy procedures $(n=11)$

\begin{tabular}{|c|c|}
\hline MALE: FEMALE & $3: 8$ \\
\hline Age range & $17-56$ years; average 31 \\
years
\end{tabular}

stapes (both anterior and posterior), stapedius tendon and long process of incus is clear and adequate enough to obviate mobilization of chorda tympani and curettage of posterosuperior canal wall in most situations. ${ }^{2}$ A little advancement of endoscope shows all these structures in a vivid and magnified way.

In the microscopic vision, the slender anterior crus of stapes often remains invisible. Moreover, higher magnification in the microscope is associated with poorer illumination, compromising the vision. These problems are not encountered with an endoscope. Extensive curettage of posterosuperior canal wall (that is often required in a microscopic procedure for proper visualization) may often give rise to retraction pocket in the long term, but this potential complication can be avoided in the endoscopic procedure. ${ }^{6}$

In microscopic procedure one can perform stapedotomy keeping the stapedius tendon and suprastructure intact, but with an endoscope, performing stapedotomy keeping all these structures intact is relatively difficult and may cause instability of long process. We encountered such situation in 1 of our patients. Once the incudostapedial joint and stapedius tendon are cut and the superstructure removed, the footplate can be seen beautifully under magnification which facilitates stapedotomy in a more precise and proper location.

At the end of 6 months follow up, we achieved a satisfactory closure of $\mathrm{AB}$ gap less than $10 \mathrm{~dB}$ in 5 patients and in between $10-20 \mathrm{~dB}$ in 4 patients. One of our patients developed sensorineural hearing loss. The reason is unknown but it appears to be due to iatrogenic damage to labyrinth during perforation of footplate. Heat generated from the tip of the endoscope as the source of light could be another potential factor as the tip of endoscope remains very close to vestibule, and could result in heat-induced damage once the footplate is perforated. ${ }^{4}$ The exposure time can also be a determining factor for the development of sensorineural hearing loss. This can be prevented by keeping the illumination lower, not keeping the endoscope for too long close to the perforated footplate, and by frequently dipping in anti-fog solution for cooling.

\section{Conclusion}

Endoscopic stapedotomy is a safe and effective approach towards stapes surgery, with better surgical field of view and minimal tissue trauma. The difficulty with one- 
handed movements can be compensated by satisfactory maneuverability with a mobile visual aid. In our small series of patients undergoing endoscopic stapedotomy, we encountered negligible difficulties in executing the surgical steps, and acceptable post-operative outcomes in terms of hearing gain.

\section{Acknowledgment}

The authors wish to thank Dr. Mainak Dutta, Clinical Tutor, and Dr. Pratyusha Sasmal, Junior Resident, Department of Otorhinolaryngology and Head-Neck Surgery, Medical College and Hospital, Kolkata, for their invaluable help and suggestions in preparing this manuscript.

\section{References}

1. Poe DS. Laser-assisted endoscopic stapedectomy: a prospective study. Laryngoscope 2000; 110: 1-37

2. Nogueira JF, Presutti L, Abreu JPS, Feijão MX, et al Endoscopic transcanal stapes surgery (stapedotomy). In: Presutti L, Marchioni D, eds. Endoscopic ear surgery: Principles, indications, and techniques, 1st ed. Stuttgart: Georg Thieme Verlag KG, 2015; p294-303

3. Pothier D. Staring off with endoscopic ear surgery. In: Presutti L, Marchioni D, eds. Endoscopic ear surgery: Principles, indications, and techniques, 1st ed. Stuttgart: Georg Thieme Verlag KG, 2015; p294-303

4. Tarabichi M. Principles of endoscopic ear surgery. In: Presutti L, Marchioni D, eds. Endoscopic ear surgery: Principles, indications, and techniques, 1st ed. Stuttgart: Georg Thieme Verlag KG, 2015; p294-303

5. Sajjadi H. Endoscopic middle ear and mastoid surgery for cholesteatoma. Iran J Otorhinolaryngol. 2013; 25: 63-70

6. Sarkar S, Banerjee S, Chakravarty S, Singh R, et al. Endoscopic stapes surgery: our experience in thirty two patients. Clin Otolaryngol 2013; 38: 157-60. 